\title{
Facile synthesis of starch-maleate monoesters from native sago starch
}

\author{
Soon Hiang Tay, Suh Cem Pang*, Suk Fun Chin \\ Department of Chemistry, Faculty of Resource Science and Technology, University Malaysia Sarawak, 94300 Kota Samarahan, Sarawak, Malaysia
}

\section{A R T I C L E I N F O}

\section{Article history:}

Received 5 September 2011

Received in revised form 23 January 2012

Accepted 25 January 2012

Available online 2 February 2012

\section{Keywords:}

Sago starch

Starch-maleate monoester

Cross-linked particles

Green synthesis

\begin{abstract}
A B S T R A C T
This paper documented a facile and green approach for synthesizing water soluble cross-linked starch-maleate monoester gel particles from native sago starch (Metroxylon sagu). Starch-maleate (SM) monoester gel was initially synthesized by reacting sago starch with maleic anhydride in an aqueous medium, and followed by precipitating in absolute ethanol. The transformation of starch-maleate gel into cross-linked gel particles of mean diameter $445 \pm 115 \mathrm{~nm}$ occurred upon UV irradiation in the presence of cerium(IV) ammonium nitrate. The substitution of maleic anhydride onto starch chains was confirmed by FTIR spectroscopy, and the degree of substitution (DS) as determined by the back-titration method was within the range of $0.03-0.21$. The water absorbency and hydrophilicity of SM samples of DS $<0.03$ was substantially lower than SM samples of DS $>0.08$. Being non-toxic, biocompatible and cheap, the potential utility of starch-maleate gel particles as drug delivery carriers in biomedical applications is therefore envisaged.
\end{abstract}

(C) 2012 Elsevier Ltd. All rights reserved.

\section{Introduction}

In recent decade, numerous biomedical applications based on the utilization of natural polymers such as proteins (e.g. collagen, gelatin and albumin) and polysaccharides (e.g. starch, dextran, cellulose and chitosan) had been reported (Elfstrand et al., 2007; Liu, Chen, Li, Hu, \& Cai, 2004; Shanbhag, Barclay, Koziara, \& Shivanand, 2007; Tiyaboonchai, 2003). Recently, starch derivatives have been studied for potential applications in the controlled release of therapeutic agents (Alias, Silva, Goni, \& Gurruchaga, 2008; Bravo-Osuna, Ferrero, \& Jiménez-Castellanos, 2008; Chen, Li, Li, \& Guo, 2007). Starch derivatives are inexpensive biomaterials which exhibit bioavailability and biocompatibility properties in biological fluid. Due to the presence of abundant and freely available hydroxyl groups on the starch chains, starch can be easily modified and tailored for various desired applications (Sun \& Sun, 2002). The hydroxyl groups of starch chains can be etherified through the formation of free radicals, or esterified with carboxylic acid, acyl chloride as well as acid anhydride (Chi et al., 2008; Fang, Fowler, Sayers, \& Williams, 2004). Various sources of starch have been utilized as the precursors for the preparation of a wide range of starch derivatives suitable for pharmaceutical applications (Elfstrand et al., 2007). Starch derivatives of high amylose maize were investigated as effective colon-targeted drug delivery carrier (Alias et al., 2008) and gastrointestinal tract drug delivery carrier (Desai, 2007). High amylose Hylon VII starch

\footnotetext{
* Corresponding author. Tel.: +60 82 583017; fax: +60 82583160.

E-mail addresses: scpang@frst.unimas.my, suhcem@gmail.com (S.C. Pang).
}

was investigated as starch excipients for transmucosal delivery (Mulhbacher, Ispas-Szabo, Ouellet, Alex, \& Mateescu, 2006). Maize starch derivative was evaluated as a potential drug delivery carrier for colon-targeting biomacromolecule drugs (Chen et al., 2007). However, little or no study has been conducted on the utilization of sago starch derivatives for biomedical applications.

In the modification and derivatization of starch, an appropriate solvent medium is essential for the effective solubilization of starch granules, and subsequent dissolution of their amylose and amylopectin components. Upon dissolution, hydroxyl groups on the polysaccharide chains become accessible to electrophillic substitution during the esterification reaction. The solubilization of starch during esterification was commonly being conducted in aprotic solvents such as $\mathrm{LiCl} /$ dimethylacetamide and dimethylsulfoxide (DMSO) (Biswas, Shogren, Kim, \& Willett, 2006; Fang et al., 2004). These solvent systems were harmful and difficult to be removed from the end products, and hence rendered them unsuitable for biomedical applications. Nevertheless, the successful starch esterification in aqueous media with a moderate degree of substitution had been reported (Xu, Miladinov, \& Hanna, 2004). It is noteworthy that sodium hydroxide is commonly being used as a catalyst in aqueous starch esterification instead of using toxic reagents such as $\mathrm{N}, \mathrm{N}$-dimethylaminopyridine, methane sulphonic acid (Biswas et al., 2006; Chi et al., 2008; Fang et al., 2004; Wang \& Wang, 2002). Enhanced starch solubility in alkaline medium had resulted in high accessibility of reagents and hence uniform substitution.

Although the preparation of starch esters with substituted hydrophobic end groups such as acetylated starch have been frequently reported (Gunaratne \& Corke, 2007; Roesser, McCarthy, Gross, \& Kaplan, 1996), little attention has been focused on starch 